\title{
Les limites du transnational comme espace de mobilisation
}

Valérie Amiraux

\section{(2) OpenEdition \\ 12 Journals}

Édition électronique

URL : http://journals.openedition.org/conflits/167

DOI : 10.4000/conflits. 167

ISSN : 1777-5345

Éditeur :

CCLS - Centre d'études sur les conflits lilberté et sécurité, L'Harmattan

Édition imprimée

Date de publication : 15 mai 1999

ISSN : 1157-996X

Référence électronique

Valérie Amiraux, "Les limites du transnational comme espace de mobilisation », Cultures \& Conflits [En ligne], 33-34 | printemps-été 1999, mis en ligne le 16 mars 2006, consulté le 30 mars 2021. URL:

http://journals.openedition.org/conflits/167 ; DOI : https://doi.org/10.4000/conflits.167

Ce document a été généré automatiquement le 30 mars 2021.

Creative Commons License 


\title{
Les limites du transnational comme espace de mobilisation
}

\author{
Valérie Amiraux
}

1 «Ici n'est pas chez vous, répéta-t-il. Le sol est à vous, oui, et si j'y posais le pied, c'est vrai que je m'égarerais sur vos terres. Mais en haut, non. Où bon me semble, je vais. » Italo Calvino, Le baron perché

Introduction

3 Longtemps donnée marginale des travaux sur l'islam implanté en Europe, le transnational est dorénavant un qualificatif à la mode pour qui s'intéresse aux identités religieuses liées aux mouvements migratoires ${ }^{1}$. . Son application dans ce champ recouvre pourtant diverses définitions et met en scène des échelles d'observation variées dans un débat scientifique aux yeux duquel le transnational fait souvent référence aux ingérences des pays d'origine dans le financement des lieux de culte bâtis en Europe ${ }^{2}$. Depuis les échanges épistolaires, le lien téléphonique, les circulations de know how à l'occasion des vacances, les retours au pays des retraités, jusqu'aux mobilisations électorales organisées à partir des pays d'accueil vers les pays d'origine, les dimensions transnationales de l'immigration sont multiples, même si leur admission comme donnée de fait passe trop souvent par l'économie d'une définition claire ${ }^{3}$. L'attribution du label "transnational » à des comportements diversifiés et surtout moins nouveaux qu'ils ne le paraissent, impose de revenir sur sa circonscription, élaborée dans cet article à partir d'une étude de cas particulière, celle de l'islam turc implanté en Allemagne ${ }^{4}$. Relativement aux utilisations du concept comme régime articulant des contraintes internationalement admises, comme circuit alternatif à l'action de l'État, voire menace à la souveraineté de celui-ci ${ }^{5}$, il nous semble en particulier nécessaire de marquer notre distance vis-à-vis d'une lecture considérant le transnational exclusivement dans une perspective relationnelle et linéaire dans laquelle l'État demeure in fine le cadre principal de référence, pour privilégier une lecture en terme d'espace. Cette hypothèse d'un « espace transnational » s'appuie sur l'analyse de trois associations islamiques turques occupant différentes positions dans le champ religieux, tant en Turquie qu'en Allemagne, quant au sens donné à 
l'appartenance et l'engagement religieux (mystique pour les Süleymanci aussi notés IKZ, étatique pour la Diyanet aussi notée DITIB, islamiste pour l'ex-Avrupa Milli Görüs Teskilatlari notée AMGT et rebaptisée depuis), et sur l'examen des pratiques spécifiques nées de leur transplantation du territoire turc sur le sol allemand, notamment partant d'exemples d'individus ayant fait le choix d'une carrière politique à partir d'une socialisation associative, dans leur pays d'origine ou sur leur lieu de résidence. La dynamique transnationale de l'islam n'est pas nouvelle même si elle se trouve valorisée de façon différente à la faveur de l'immigration vers l'Europe, l'appartenance à la Umma, la communauté des croyants, n'étant pas structurée par un principe géographique territorial ${ }^{7}$. La pertinence tient dans notre étude de cas à l'idée que des réseaux de jeunes musulmans turcs, sédentarisés en Allemagne tirent avantage, notamment en matière de représentation politique, de leur environnement bien que n'étant pas citoyens de la République Fédérale. Les associations islamiques - c'est-àdire militant pour la reconnaissance et la défense des droits des musulmans - dans lesquelles ils choisissent de s'engager comme sympathisants, clients, militants ou leaders, offrent des formes de participation alternatives à la seule position citoyenne, atouts d'autant plus considérables qu'ils sont mis en scène dans un contexte offrant aux producteurs de référents religieux une stabilité, une longévité, une autonomie et une indépendance radicalement nouvelles et différentes au regard de celles liées à l'environnement turc ${ }^{8}$. La capitalisation des ressources par le groupe comme par l'individu se trouve donc facilitée par le contexte. Temporairement déconnectés de la souveraineté nationale, les acteurs organisent les voies et les moyens de leur participation par d'autres canaux que celui de la citoyenneté9. Revenir sur les écarts entre, d'un côté les logiques produites par les normes et les institutions de part et d'autre des flux migratoires - c'est-à-dire en Allemagne la loi sur la nationalité, en Turquie l'articulation complexe entre islamisme et sécularisme - et, de l'autre le fonctionnement, les trajectoires et stratégies des musulmans vivant en Allemagne, met en scène ce que nous désignons comme un «espace transnational» favorisant la capitalisation d'un certain nombre de ressources, matérielles ou symboliques. L'articulation entre opportunités et besoins, mobilisation et participation, revendication et exclusion, particulièrement visible dans la restitution d'éléments biographiques de certains de nos interlocuteurs, illustre les étapes de la construction de cet espace transnational, et permet de revenir sur ses " conditions de production ", de souligner la pertinence de l'adéquation entre un contexte et certains types de revendications (notamment sur comment se fait le travail de mobilisation, entre une plus grande visibilité politique de l'islam en Turquie et un fort mouvement de privatisation du religieux en Allemagne), enfin de s'arrêter sur les limites de la reconversion de certains capitaux.

4 Le transnational, de la relation à l'espace De la même façon que l'on désigne comme diaspora toute communauté dispersée consciente de cette situation et s'énonçant comme telle, la qualification de transnational permet rarement de déterminer s'il s'agit d'une relation bilatérale, transfrontalière ou diasporique, et ajoute au flou du label ${ }^{10}$. Ouvert sous la houlette des spécialistes de relations internationales depuis les années soixante-dix, le débat sur le transnational est trop souvent réduit au statut d'alternative aux théories stato-centrées, signant la fin de l'hégémonie de la géopolitique classique, négligeant par exemple d'observer les positionnements individuels dans différents espaces nationaux, exploitant la dimension transnationale comme une ressource au service d'une stratégie ${ }^{11}$. De facto, les flux migratoires 
traversent des espaces nationaux, font passer des individus, des groupes, des familles, des enjeux, d'un lieu d'origine à un pays dit d'accueil. En sus de cette dimension factuelle se révèle, quelques décennies après la signature des contrats de main d'œuvre en Europe, l'importance de la dimension réticulaire. Confirmant l'assertion selon laquelle ce ne sont pas seulement des individus mais des réseaux qui migrent ${ }^{12}$, ce mode d'organisation est une clef essentielle de la compréhension du phénomène transnational, en termes de diffusion de l'information, de communication, de circulation des hommes, des biens, des idées, de capitalisation et de mobilisation des ressources. Les contacts, coalitions et interactions croisant les frontières étatiques, non contrôlés par les organes centraux de politique étrangère des différents gouvernements, occupent donc une place centrale dans ces dynamiques, permettant notamment de faire jouer la ressource «solidarité » comme ce fut le cas au sein de la population turque musulmane en Europe lors du conflit bosniaque ${ }^{13}$. Dans le cas des Turcs en Allemagne, et plus largement des Turcs en Europe, l'implantation des individus, le continuum des entrées puis le regroupement des familles s'inscrivent dès le début de leur présence, dans les années soixante, dans une dynamique de réseaux claniques, familiaux, villageois ${ }^{14}$. Les réseaux et les formes d'organisation non étatiques sont autant d'acteurs clefs des pratiques transnationales, tant à l'échelle collective qu'individuelle, notamment car ils offrent une alternative en termes de prestations, voire jouissent d'une certaine efficacité régulatrice ${ }^{15}$. De la même manière, se désigner comme musulman ne correspond pas seulement à l'inscription dans une lignée de croyants ${ }^{16}$, mais aussi à une position d'acteur socialisé dans un réseau particulier. L'utilisation du terme « transnational » impose quelques éléments de clarification et de précision, notamment au regard de son utilisation, tant par les spécialistes de relations internationales pour qualifier l'évolution d'une vision du monde stato-centrée vers une réalité multicentrée ${ }^{17}$, la complexification des affaires étatiques et l'émergence d'acteurs non-étatiques sur la scène internationale, que par les théoriciens des mouvements sociaux et plus récemment par les spécialistes de l'immigration. Il correspond avant tout à un constat, celui de la moindre pertinence du prisme étatique pour lire les relations internationales. Parallèlement, il dérive aussi de l'entrée en scène de nouveaux modes de communication, des changements dans le rapport au temps, à la distance, de la puissance des médias, et signe le dépassement du territoire et de ses frontières comme références. Enfin, le transnational - au sens des connexions qui ont été élaborées hors cadre étatique par des groupes secondaires et dans un espace échappant au contrôle direct des autorités turque ou allemande - se présente en soi comme une ressource démontrant que les pratiques politiques n'ont pas nécessairement besoin d'être actionnées depuis une base territoriale nationale pour être efficaces. Le 24 décembre 1995, le nombre de voyages organisés par l'Avrupa Milli Görüs Teskilatlari depuis les grandes villes d'Allemagne (Berlin, Cologne, Stuttgart, Munich) à destination de la Turquie pour permettre aux Turcs d'Allemagne de participer au scrutin législatif et d'apporter leurs voix au Refah, témoignait de la réussite de l'entreprise et du lien quasi indéfectible entre les Turcs d'Allemagne et la politique de leur pays d'origine ${ }^{18}$. Dans le contexte post-migratoire de l'Europe occidentale, les espaces nationaux perdent en outre de leur évidence : on ne réside pas forcément dans l'espace de sa participation politique citoyenne. Le mouvement d'implantation dans les sociétés civiles et politiques d'accueil, conséquence de la transition de la migration de travail à l'installation familiale ajoute ainsi à la spécificité de la configuration Allemagne-Turquie. Les associations gestionnaires de l'islam se 
trouvent de fait classiquement confrontées à une série de problèmes générationnels, d'absence d'une autorité légitime capable d'énoncer la norme religieuse dans le pays d'accueil, et à la contrainte de produire ses propres institutions. Plus qu'un territoire qui s'effacerait, c'est l'exercice de la souveraineté de l'État sur celui-ci qui se trouve modifié et reformulé par effet de concurrence avec des acteurs «libres de souveraineté » ${ }^{19}$. A propos de ces réseaux transnationaux, T. Risse souligne leur caractère non-étatique, en prenant soin de rappeler qu'il ne signifie pas pour autant le retrait de l'État comme acteur ni comme variable déterminante dans la constitution de réseaux d'acteurs transnationaux ${ }^{20}$. La matrice religieuse de l'islam turc implanté en Allemagne confirme l'importance de cette remarque. Contournant l'État turc et sa gestion laïque du religieux, les réseaux associatifs turco-islamiques n'en sont pas moins à l'origine de son installation à Berlin au début des années quatre-vingt, l'obligeant comme l'atteste l'entrée en scène de la DITIB à se positionner dans l'espace migratoire suivant une stratégie de maintien des cercles de son influence hors du territoire légitime d'action ${ }^{21}$. Sur le plan de la gestion des affaires religieuses en Allemagne, l'État turc est ainsi contraint de prendre position dans le champ migratoire, de se doter des moyens d'accès à un territoire non strictement national, et de devenir lui aussi acteur du transnational. L'intervention d'Ankara dans la vie religieuse de ses ressortissants installés en Allemagne fait également suite au coup d'État du 12 septembre 1980, ayant frappé d'interdiction, avec d'autres, le parti de Necmettin Erbakan ancien leader du Refah Partisi-Parti de la Prospérité ${ }^{22}$, et chef du gouvernement de la coalition Refahyol (Refah Partisi-Doğru Yol Partisi) entre juin 1996 et juin 1997. Cette reprise en main du religieux participe donc d'une double logique, gouvernée d'une part par l'agenda de politique intérieure turque (l'État délocalise son action au plan religieux), répondant de l'autre à une logique de situation ponctuelle, liée à un contexte spécifique et au besoin de prendre position en Allemagne face à ce qui est considéré comme une déviance à l'islam orthodoxe turc tel qu'institué légitime par le dogme séculaire construit par les élites kémalistes ${ }^{23}$. L'État turc tente alors de reprendre position dans un territoire dont il n'est pas le souverain légitime ${ }^{24}$. C'est à ce niveau du raisonnement que parler d'espace plutôt que de relation prend son sens. Dans ce cas précis, cela revient donc à identifier une forme, des structures, un ensemble de fonctions, id est «l'espace de la pratique sociale, - celui qu'occupent les phénomènes sensibles, sans exclure l'imaginaire, les projets et projections, les symboles, les utopies $"{ }^{25}$. Cette idée d'espace transnational évite l'écueil d'une perception exclusivement bilatérale et permet de suivre la production de pratiques dans différents environnements nationaux, leur déterritorialisation à la faveur de la migration et leur institutionnalisation, voire leur incapacité à sortir de cette configuration originale. Elle permet alors d'envisager les processus sociaux dans leur coexistence et leur simultanéité. Ce dernier point est d'intérêt notable dans la mesure où ces pratiques issues de l'espace transnational ne sont pas exclusivement dévolues à l'économique mais se poursuivent, se perfectionnent et se professionnalisent même dans le cadre de carrières politiques. Ainsi, tandis que le séjour en Europe produit de nouvelles façons d'être musulmans, la variable générationnelle pertinente au regard de la redéfinition de l'appartenance religieuse, non plus tant héritée que conquise, transformée et réinventée, n'amenuise ni ne dévalorise l'espace transnational, mais y puise même les ressources nécessaires à l'accès à la représentation politique. La notion de "déterritorialisation " ou plutôt l'idée d'une nouvelle contiguïté des territoires ${ }^{26}$ devient dès lors la pierre angulaire de la réflexion sur le transnational, sur ses modes de fonctionnement et ses limites, face à 
un État entendu comme exerçant sa souveraineté sur un territoire donné, et lié dans sa légitimité et son identité à cette contrainte ${ }^{27}$. Le transnational, dans notre étude de cas, ne signe pas la fin d'une définition territoriale de l'action de l'État, mais en reformule le mode d'exécution, ou plutôt lui adjoint des alternatives inédites, notamment en matière de redistribution, revalorisant en particulier les dimensions locale et transfrontalière. Ce mouvement est particulièrement perceptible, d'une part dans les thèmes de travail investis par les associations islamiques (drogue, aide au devoir, sport...) soucieuses de se construire une notabilité de quartier, d'autre part dans la recherche d'une performance, d'une efficacité maximale des activités entreprises plus que d'une quête de légitimité ${ }^{28}$. Le transnational favorise en outre l'extension d'affiliations identitaires au-delà des frontières nationales, dans des espaces sociaux aux frontières non plus ancrées dans le principe de territorialité et loin de l'idéal contractuel sans intermédiation liant exclusivement le citoyen à la Nation ${ }^{29}$. Il valorise donc ponctuellement certains cadres d'opportunité au détriment d'autres, en fonction des besoins des acteurs, au moment où s'expriment les revendications, indépendamment de l'institution de la citoyenneté. Il se manifeste notamment dans les dynamiques réticulaires, le recyclage de certaines ressources, mais toujours comme un espace quasi informel dont les contours à géométrie variable sont indexés sur la perception qu'en ont les acteurs. La multiplication et la diversification des identifications et des appartenances permettent non seulement à des acteurs nonétatiques de se positionner comme structures d'autorité alternatives au plan international, faisant ainsi concurrence au droit exclusif des gouvernements à le faire, mais sont, de surcroît, à l'origine d'effets de composition résultant de l'agrégation des actions individuelles et collectives de ces nouveaux acteurs.

5 Une convergence heureuse de contraintes et d'opportunités qui font du transnational une ressource L'espace transnational est aussi le produit d'un effet de composition né de la coïncidence des intérêts des deux États, allemand d'une part, turc d'autre part, dans la gestion de l'islam. Cette conjonction est à envisager dans la perspective de séries d'opportunités, extérieures au groupe d'acteurs associatifs et offertes par l'un et l'autre des contextes. On ne migre pas vierge de toute expérience ou de culture politiques mais bien porteur de représentations, symboles et stocks cognitifs ${ }^{30}$, notamment en référence à l'histoire du face-à-face séculaire État/Religion ${ }^{31}$. L'espace transnational s'avère ainsi essentiellement le produit de la rencontre entre une population particulière, musulmane et turque, issue d'un contexte véritablement inhibé en matière de religion, et une série d'opportunités proposées par le contexte allemand ${ }^{32}$. Sa mise en place se fait sur le mode de l'importation des répertoires d'action, avec ou sans l'aval de l'État d'origine, du maintien d'une sensibilité à la vie politique du pays d'origine notamment par l'intermédiaire des médias ${ }^{33}$. Il faut de surcroît ajouter à ces remarques le rôle des experts, non des moindres dans cette construction, en particulier au regard de la convergence de vues entre gestion politique et lecture scientifique des problématiques de l'immigration en général et de l'islam en particulier ${ }^{34}$. L'interaction de ces différentes variables héritées des deux contextes peut être expliquée en termes d'opportunités dont l'acteur, individuel ou associatif se saisit en fonction de la valorisation de ses intérêts. Il en est ainsi de l'articulation entre les différentes structures intérieures (i.e. la façon dont l'État est organisé) et le degré d'institutionnalisation dans le traitement de la présence de l'islam en Allemagne. Le fédéralisme et ses «many alternative pockets for participation " ${ }^{35}$, la fragmentation des niveaux de décision, l'organisation corporatiste des institutions, celle de la société 
civile, le partenariat État-Religion, sont autant d'éléments qui entrent en compte dans le dynamisme de l'espace transnational et orientent les acteurs dans leur choix ${ }^{36}$. Ainsi, la possibilité de se constituer en association d'étrangers (eingetragene Verein) permetelle aux différents acteurs de l'islam turc, sanctionnés, voire censurés à certains moments de l'histoire récente de la Turquie, d'acquérir un statut et une visibilité dont ils ne sont pas forcément détenteurs dans le pays d'origine. A bien des égards, la présence de l'islam en Europe peut être dit "path dependent $"{ }^{37}$ dans la mesure où étudier les enjeux liés à sa présence en Europe revient pour l'essentiel à retracer le cheminement du lien entre politique et religieux, c'est-à-dire à évaluer l'intégrabilité de l'islam à l'aune de son potentiel d'institutionnalisation, renvoyant les États européens à leur histoire religieuse récente ${ }^{38}$. Cette idée s'applique aussi bien aux contextes d'origine qu'à ceux des pays dits d'accueil. Ainsi, la sécularisation considérée comme le transfert de fonctions d'intérêt général de la tutelle religieuse à l'administration laïque (État séculier) - ne correspond-elle pas à une réalité similaire en Turquie et en Allemagne. Tandis qu'en Turquie elle débouche sur la promotion concurrente de deux systèmes de valeurs antagonistes -islamisme versus kémalisme -, l'histoire allemande révèle un processus de sécularisation dans lequel la rivalité confessionnelle entre Catholiques et Protestants se traduit politiquement par la reconnaissance institutionnelle de la parité dont la Paix d'Augsbourg et les Traités de Westphalie ont fixé les premiers jalons. Ce partenariat entre État et Église prend toute son importance dans la démarche exprimée par les associations islamiques depuis 1977, date à partir de laquelle différents responsables associatifs, des fédérations, déposent régulièrement des demandes de reconnaissance officielle par les pouvoirs publics, sans succès jusqu'ici au motif qu'il n'existe aucune structure fédérant suffisamment d'intérêts pour être désignée comme représentant tous les musulmans. L'hostilité des cadres juridique et politique à l'égard de l'islam en Turquie se voit en quelque sorte compensée par les avantages et opportunités fournies, proposées par l'architecture juridique allemande. «Je tiens pour une chance la relation de partenariat entre l'État et l'Eglise dans l'histoire de notre République, et nous devrions tout faire pour la maintenir en l'état ", déclarait en 1997 le Chancelier Kohl ${ }^{39}$. Issues d'une République dont la laïcité s'est construite sur la base du contrôle de la religion et de son exploitation à des fins politiques plus que sur son émancipation et son indépendance des structures de l'État, les associations islamiques transplantées en Allemagne y font l'expérience de la liberté d'organisation et de la reconnaissance publique de la légitimité d'un partenariat traduit dans le statut juridique de Corporation de droit public (Körperschaft des öffentlichen Rechts). Outre l'argument financier concernant l'impôt d'église, la corporation de droit public décide seule du contenu des cours de religion, de l'ouverture d'écoles confessionnelles et de lieux de culte. Elle gère également seule les directeurs de conscience (Seelsorge) dont la présence est autorisée dans nombre de services publics (armée, hôpitaux, police, prisons), et enfin bénéficie d'un droit de passage gratuit (publicité) sur les télévisions et radios publiques. L'intérêt matériel et symbolique de ce statut est à l'origine de la stabilité de cette revendication, plus une constante qu'une phase parmi d'autres, dans l'évolution des demandes de la part des associations islamiques en Allemagne. Dès lors, nous retenons deux dimensions du concept de structure d'opportunité politique : en premier lieu l'idée que les éléments de la structure politique sont déterminants non dans le sens d'une soumission aux structures, mais d'une exploitation de celles-ci ; en second lieu, que les facilités d'accès à la participation politique apparaissent fondamentales dans la 
mobilisation des ressources par les réseaux comme dans les positionnements individuels. Libres de toutes contraintes institutionnelles centralisatrices du type «Eglise » pour ce qui concerne notamment la gestion des pratiques, les associations islamiques turques installées en Allemagne déploient une logique entrepreneuriale dans l'établissement d'un lien entre la capacité de s'adapter aux opportunités et leurs propres objectifs de reconnaissance. La légèreté des structures permet de mêler activités sociales, économiques et par la suite de rendre pertinente une visibilité politique de ces intérêts identitaires religieux. Dans le cas des groupes turcoislamiques, l'évitement de l'intervention de l'État turc est la clef des choix des modes d'action et d'organisation. Sur plusieurs points, notamment en matière d'éducation religieuse, les autorités allemandes, régionales et fédérales, ont, à partir des années quatre-vingt, choisi de collaborer avec le gouvernement turc et le Bureau des Affaires Religieuses (la DITIB en Allemagne), trouvant dans cette gestion laïque une alliée de choix. Troisième confession de l'Allemagne réunifiée, l'islam est donc géré de l'extérieur, sur un "mode diplomatique " ${ }^{40}$, comme une collaboration inter-étatique relevant des Affaires Etrangères plus que de l'Intérieur, en parfaite coïncidence avec la terminologie particulière de l'Allemagne en matière d'immigration ${ }^{41}$. Le séjour des Gastarbeiter (travailleurs-hôtes) étant conçu comme provisoire, les revendications confessionnelles des ausländische Mitbürger (concitoyens étrangers) ne s'inscrivent pas sur l'agenda de politique intérieure. Le choix de faire de la DITIB, symbole de l'expérience turque de laïcité et de la convergence des intérêts des deux États, un interlocuteur officiel pour les affaires islamiques atteste de la volonté allemande de gérer la présence de l'islam sur son sol comme une affaire de politique étrangère, et de la maintenir dans l'espace de politique intérieure turque, en tout cas hors de portée des sphères d'intervention légitimes de l'Allemagne (participation politique via les naturalisations et reconnaissance de statut par le droit). L'inscription sur l'agenda politique n'intervient ainsi que par l'intermédiation des questions de sécurité, de défense et d'affaires étrangères. Du côté des acteurs de l'espace transnational, la dimension sécuritaire a longtemps conduit à la négation de cette ressource, dénoncée par leurs adversaires politiques en Turquie, et surveillée par les autorités allemandes. Elle a été plus particulièrement et franchement assumée lorsque le Refah est arrivé à la tête de la coalition gouvernementale formée avec le DYP en juin 1996, au point de figurer dans la liste des mesures sur lesquelles le Conseil National de Sécurité turc demandait au gouvernement dirigé par N. Erbakan de s'engager en février 199742. La mise en acte du transnational se traduit par une circulation des idées, des hommes et des biens entre filiales allemandes délocalisées et partis ou formations d'origine en Turquie, mais s'élabore aussi en relation avec les enjeux de l'immigration, et avec le fait que les associations religieuses doivent leur survie à un certain nombre de performances ${ }^{43}$, à leur capacité à fournir services sociaux, confort et soutien, à faciliter le quotidien, à maintenir un lien, à assurer la production de références culturelles à une population rajeunie. Pour ce qui concerne la gestion immédiate de la vie religieuse en Allemagne, les cadres de l'action produits par le contexte immédiat ont contribué à la mise en place de mécanismes de compensation notamment dans la mise en scène des compétitions entre associations rivales, et ont même conduit à une répartition du marché entre organisations concurrentes. Le partage d'une expérience commune, celle de la migration, et les mobilisations pour la défense des intérêts des acteurs, n'impliquent pas d'agir ensemble, mais valorisent plutôt des logiques sectorielles. C'est principalement sur le terrain, de manière très concrète, que se matérialisent les 
oppositions entre le gouvernement et les groupes islamiques du type IKZ ou AMGT, la concurrence la plus acharnée se livrant notamment sur la question de la nomination des imams - lesquels, en Allemagne se mettent au service d'une clientèle et non plus de l'État - et de la construction des lieux de culte. La nationalité fermée et la réglementation juridique des liens entre État et Église ont en fait contribué à maintenir la légitimité du transnational comme mode d'action, ressource et forme de communication, en permettant l'accès, à l'intérieur d'un territoire donné, à un "sens de la communauté ». Sur ces opportunités juridiques, options politiques et cadres discursifs, se greffent l'indifférence et les partis pris de l'université vis-à-vis de l'islam installé sur le sol allemand ${ }^{44}$ l'absence d'études statistiques au niveau fédéral ${ }^{45}$, négligence scientifique et désintérêt politique se nourrissant l'un l'autre. Le thème «islam en Allemagne » est littéralement squatté par les orientalistes, les journalistes, les "praticiens" (travailleurs sociaux, syndicats, Eglises), des acteurs partiaux, et quelques rares approches de sciences sociales, massivement monographiques et descriptives ${ }^{46}$. La relative indifférence de la recherche, ou plutôt le cantonnement des investigations sur l'islam en Allemagne à certaines options disciplinaires, se distingue de la situation française où la construction de la pression politique (growing reality pressure) autour de l'islam comme menace et danger, aussi bien en France qu'hors de France, a créé un rapport d'offre et de demande entre politique et recherche scientifique. Cette dynamique n'existe pas en Allemagne ou, en tout cas ne traverse pas l'opinion publique avec la même sensibilité, en particulier parce que les jeunes Turcs nés et vivant en Allemagne n'ont jamais été perçus comme une clientèle électorale ${ }^{47}$. De contrainte et fatalité liées à la déterritorialisation par le mouvement migratoire, le transnational devient donc pratiquement un espace de construction de liens, de sens, d'identité, dont le religieux, dans un premier temps contraint d'y évoluer, tente de s'accommoder en y puisant des ressources, gagnant en autonomie et en mobilité. Au plan associatif, d'un côté, indexés sur le pays d'origine, ce sont les projets politiques qui alimentent l'islamisme turc, qu'il s'agisse des Süleymanci ou de Milli Görüs et même de la Diyanet dans ses tentatives de briser l'ascension de ses rivales. De l'autre, sur un plan culturel, le discours associatif se construit à partir d'une demande, celle d'une clientèle de jeunes croyants nés et socialisés en Allemagne dont les projets de vie sont davantage liés à leur lieu de résidence qu'au lieu de naissance de leurs parents. Maintenir la lignée, la recréer, qu'il s'agisse de la transmission des pratiques ou de la réappropriation d'un corpus livresque et gestuel ${ }^{48}$, prend alors une importance capitale dans un contexte où le pluralisme devient norme sociale. Le processus de construction de la lignée croyante engage «la matérialité de la continuité du groupe (l'existence concrète de celui-ci dans la durée), la stabilisation du croire dans le temps (sous forme de croyances, de pratiques, de valeurs partagées, de normes de comportement, de coutumes, etc.) et l'inscription symbolique de la permanence du groupe sur la scène sociale (...). » ${ }^{49}$. Les quatre logiques - communautaire, émotionnelle, éthique, culturelle - de ce processus se retrouvent sectorielles et générationnelles dans le champ migratoire, accentuant la fonction primordiale de médiateurs attribuée aux associations. Le déplacement de l'islam des pays d'origine vers les pays d'accueil modifie en outre le rapport du croyant à sa communauté d'appartenance à différents niveaux. Lorsqu'une institution existe, comme dans le cas de la Turquie et du Bureau des Affaires Religieuses, c'est le rapport de l'individu croyant à celle-ci, à sa force symbolique et à son autorité, qui s'en trouve affecté, davantage caractérisé par l'autonomie de celui-ci, redevenant en quelque sorte protagoniste de sa propre foi. Les 
travaux récents sur l'islam en Europe en font unanimement état, quel que soit le pays concerné, parlant notamment de subjectivation, d'individuation ${ }^{50}$. Le phénomène n'est pas nouveau, mais dans le cas de l'islam, religion minoritaire, ce mouvement est affecté d'une autre dimension liée à la localisation en Europe et à «l'absence d'autorités religieuses islamiques légitimes dans les pays d'accueil qui puissent dire ce qu'est la norme, et enfin l'impossibilité d'une coercition juridique tout autant que social, communautaire et coutumière, qui inscrive la pratique religieuse dans l'ordre de l'évidence et du conformisme social. La religiosité doit donc s'éprouver comme choix et comme foi ${ }^{51}$. Ce processus d'individuation va de pair avec le mouvement plus général de sécularisation - c'est-à-dire d'un changement de place de la religion qui ne perd pas pour autant sa valeur en termes d'explication du monde - certains parlant même d'un islam sécularisés ${ }^{5}$. Ces phénomènes d'hybridation, de créolisation pour reprendre le terme proposé par U. Hannerz ${ }^{53}$ bénéficient de la dimension transnationale, de la possibilité d'une inscription individuelle dans plusieurs espaces, en même temps qu'ils tirent profit du contexte de sécularisation ${ }^{54}$. Ces processus d'hybridation sont à la fois cause et conséquence des mécanismes transnationaux: "Solidarités religieuses, régionales, culturelles, linguistiques et économiques inscrivent concurremment l'individu dans des espaces multiples dont l'équilibre reste fonction de l'intensité de chacune des allégeances ainsi consenties. " ${ }^{55}$. L'implication de l'acteur dans une pluralité de dynamiques réticulaires offrant diverses formes de redistribution des ressources traduit un pluralisme à l'échelle de l'individu dont la réintégration dans un système politique national ne se fait pas sans difficulté. Les segments de biographies que nous proposons dans la dernière partie de cet article permettent de prendre en considération cette polychromie, la différenciation des appartenances et des soussystèmes dans lesquels les individus évoluent, faisant le lien entre Turquie et Allemagne à différents niveaux (allégeance politique, investissements économiques, stratégies matrimoniales, communications). Certaines pratiques, étiquettes, statuts ne franchissent pourtant pas la "frontière", notamment en relation avec des exigences d'invisibilité différentes, face à des modalités de contrôle social exercées différemment d'une société à l'autre.

$6 \quad$ Les limites de la ressource transnationale Les itinéraires individuels, tout comme les offres à caractère communautaire fondées sur l'appartenance religieuse, proposées par les associations islamiques en Allemagne, s'inscrivent dans un espace social que nous avons défini comme transnational, consistant en un « interplay of their social positions in two different societies and is characterised by a severe deficit in symbolic and identity capital in regard to both societies " ${ }^{56}$. Ce jeu entre-deux ne connait pas de restriction quant au secteur dans lequel il est élaboré et peut concerner des activités entrepreneuriales classiques, culturelles, religieuses, ce que l'on a appelé l'ethnic business ${ }^{57}$, mais également se traduire par un investissement professionnel dans une carrière politique, même si cela demeure le fait d'une minorité. La performance et l'efficacité de l'espace transnational sont évaluées par les formations politiques turques à l'aune de paramètres simples dont les récoltes de fonds et la mobilisation des électeurs sont les deux indices principaux. La captation des voix de l'étranger passe par une présence constante et régulière de personnalités du Refah dans les activités de l'AMGT. Premiers indices, les visites régulières de Necmettin Erbakan aux Turcs d'Allemagne depuis 1975 remplissent une fonction intégrative indéniable, tant à destination des fidèles qu'à celles des responsables. Pour bon nombre de nominations à des postes-clefs de la hiérarchie Milli Görüs, l'autorité vient de Turquie ${ }^{58}$. Les objectifs 
principaux sont majoritairement financiers, et les mesures prises à cet égard sont efficaces. En 1991, on estimait à six millions de DM la somme d'argent passée d'Allemagne en Turquie pour soutenir la campagne électorale du Refah ${ }^{59}$. Les mesures prises par Erbakan, à peine arrivé au pouvoir, montrent un peu plus encore la clientélisation entreprise auprès des Turcs d'Europe, même si ses effets sont peu significatifs et ne remportent pas le succès escompté. Cuvrer depuis l'Allemagne permet donc d'échapper au contrôle de l'État sans pour autant s'extraire de thématiques nationales. Dans ce sens, la transnationalité n'exclut ni la pression sur, ni le combat contre un système étatique jugé illégitime et dont l'action des Süleymanci et de Milli Görüs, pour ne pas dire leur existence, sont aussi les produits. Il n'est ainsi pas exclu que l'interdiction du Refah ait permis à l'AMGT de capitaliser en Allemagne les effets d'une exclusion de la participation politique en Turquie, trouvant là une nouvelle ressource, la possibilité de mettre en œuvre un nouveau répertoire de recrutement ${ }^{60} . \mathrm{Z}$. est turc et s'est toujours refusé à prendre la nationalité allemande. Chef d'une entreprise florissante installée pour partie en Turquie, pour partie en Allemagne, il est élu en janvier 1990 représentant des étrangers de la commune de Hanovre sur une liste d'Union islamique rassemblant différents courants et nationalités, l'année même de son élection à la Chambre de Commerce et d'Industrie de la région. Défendant les intérêts de ses coreligionnaires en Allemagne et soutenant financièrement l'initiative de Cindoruk et I. Sezgin lors de leur départ du DYP alors en coalition avec le Refah de N. Erbakan $^{61}$, Z. mène sa vie sur deux territoires, passant $50 \%$ de son temps entre Allemagne et Turquie, s'affichant comme joyeux divorcé de son épouse allemande en Allemagne avant d'épouser son actuelle épouse turque à Istanbul. Aucun lien entre ces deux espaces d'activité si ce n'est lui et un attachement viscéral à Trabzon, sa ville d'origine, dont il représente les intérêts footballistiques en Allemagne et pour laquelle il décroche des crédits publics en Turquie. E., n'en déplaise à l'administration et à la juridiction allemandes, possède la double-nationalité, turque et allemande. Engagé dans l'Avrupa Milli Görüs Teskilatlari depuis près de dix ans lorsque nous le rencontrons en $1993^{62}$, il milite en même temps dans la section CDU de son quartier de résidence berlinois, Kreuzberg. En septembre-octobre 1997, alors qu'il vient d'être désigné comme délégué au Landtag (assemblée fédérale) de la CDU pour la ville de Berlin, la dénonciation de son passé de militant "islamiste » par un Turc "laïc », lui aussi militant de la CDU, met fin à cette brillante ascension dans l'une des institutions clefs de la République Fédérale. Osman Y. et Ali Y., respectivement ancien Président et Secrétaire Général de l'AMGT, ont quant à eux tous deux tenté leur chance aux législatives de décembre 1995, sous la bannière du Refah Partisi de Necmettin Erbakan. Si le premier obtient son fauteuil de député sans difficulté, le second essuie un cuisant échec au terme d'une campagne de presse dénonçant le titre dont son passé de Turc d'Allemagne. Dans un premier cas, il s'agit d'un engagement qui prolonge une ligne communautaire et repose sur un charisme situationnel lié à la réussite du businessman qui n'a pas perdu de vue ses origines. Si la mobilisation se fonde en Allemagne sur la carte « islam », elle s'en détache complètement en Turquie, voire s'y oppose. Dans les deux cas, le statut économique de $\mathrm{Z}$. constitue la ressource principale. Le deuxième profil fait pour ainsi dire «le choix de l'Allemagne », dans la mesure où, quittant la seule représentation communautaire, l'acteur prend la nationalité allemande et entre dans une formation partisane. La troisième catégorie concerne des interlocuteurs qui réussissent ou échouent dans leur tentative de rentrer en Turquie dans le cadre d'une élection, municipale ou législative. Ces itinéraires, minoritaires au regard de l'ensemble 
de la population d'origine turque vivant en Allemagne, différemment profilés, représentent trois options différentes dans le choix du politique. Le contexte allemand a valorisé la mise en acte de ces choix en faveur du politique, souvent élaboré dans un premier temps sur le terreau communautaire avant, dans un second temps, de changer d'échelle (locale, nationale) sans pour autant perdre de vue l'intérêt du groupe d'origine. Les Conseils d'étrangers (Ausländerbeiräte) et les Conseils d'Entreprise (Betriebsräte) sont souvent les premières arènes dans lesquelles s'élabore ce type de parcours, même s'il s'agit - en tout cas pour les Conseils d'étrangers municipaux avant tout d'un travail informatif et consultatif ${ }^{63}$. Qu'il s'agisse de se faire élire en Turquie ou d'œuvrer en Allemagne, le choix du politique repose sur une capitalisation de ressources en tout genre, mixe de statut social, de positions économiques, de contrôle sur les sources d'informations, de popularité/réputation ${ }^{64}$. Sortant des structures associatives comme cadre exclusif de leur engagement, les individus entrés en politique à partir d'une expérience "allemande» de socialisation militante islamique jouent sur la transition entre les mobilisations de type communautaire et une participation citoyenne turque ou allemande. Ces initiatives, ponctuelles et minoritaires, construisent des modes d'entrée variés dans les espaces politiques locaux ou nationaux et se distinguent les unes des autres par le choix du territoire d'exercice de la représentation. Toutefois, qu'il s'agisse d'une clientélisation communautaire dans un cadre local, $\mathrm{du}$ " choix de l'Allemagne » via les formations politiques fédérales, ou $\mathrm{du}$ « retour au pays", les acteurs capitalisent ressources et capitaux dans un espace transnational, produit d'une configuration post-migratoire particulière. Se pose alors la question de la convertibilité des gains liés à une capitalisation de ressources dans un espace d'énonciation différent de celui dans lequel se réalise l'accumulation : peut-on se faire élire en Turquie sur la base d'une biographie de Turc d'Allemagne et, inversement, peut-on se construire une position de représentant sans sacrifier tout particularisme au nom de l'universel ? Ces questions posent in fine celle des limites de la conversion des ressources d'une perspective transnationale à un cadre national. La réintégration de l'espace social turc que signe l'engagement dans une dynamique de représentation politique ${ }^{65}$, qu'elle soit locale ou nationale, révèle les distorsions entre statut social, capital de reconnaissance et les coefficients de légitimité dont sont affectés ces marqueurs ${ }^{66}$, et traduit les limites d'une lecture strictement instrumentale des mobilisations entendues au sens de processus par lequel une unité sociale prend le contrôle de certaines ressources ${ }^{67}$. La mobilisation politique de ces acteurs, individuels et collectifs, bloque dans le processus de retour au territoire d'énonciation premier, non pas en relation avec une contestation frontale du principe territorial - i.e. caractéristique du mode étatique de gestion du politique dans lequel les frontières légitimes servent de fondement à l'exercice de l'autorité -, mais du fait de leur émancipation de ce cadre de référence strict au profit d'une logique d'identification religieuse. Les ressources ne sont pas des biens matériels opérant hors de toute logique de rapports. De la même manière, la mobilité économique et la moindre différence entre Allemands et Turcs sur le plan matériel ne drainent pas, en terme d'identification du groupe dans l'espace social, les capitaux culturels et symboliques - i.e. ceux dont disposent ceux ayant obtenu suffisamment de reconnaissance pour être en position d'en imposer à d'autres - permettant une totale liberté de mouvement ${ }^{68}$. Dans le même ordre d'idée, lexiques et répertoires ne conservent pas une valeur identique selon le territoire d'application. Si certains anciens de Milli Görüs sont aujourd'hui maires ou députés en Turquie, l'exploitation du ticket transnational n'est donc pas toujours 
rentabilisé. Ali Y. échoue ainsi dans sa tentative de devenir député du Refah précisément en relation avec le changement de valeur d'un statut dont il est titulaire en Allemagne et que l'espace public turc juge anticonstitutionnel. Installé en Allemagne depuis 1978 après un cursus universitaire de théologie qui le conduit à exercer comme mufti, Ali Y. devient Secrétaire Général de l'AMGT en 1985 puis Président de l'Europäische Moscheebau - und Unterstützungs Gemeinschaft (EMUG), filière immobilière de la Islamische Gesellschaft Milli Görüs, nouveau nom de l'AMGT. En même temps qu'Osman 0 . et d'autres, il fait partie de la vague de candidats transférés par N. Erbakan sur les listes électorales des législatives de 1995. La défaite d'Ali Y. est directement liée à une polémique déclenchée par la révélation dans la presse de sa position de Scheikh ül Islam de l'Islamrat (Conseil islamique), organisation fédérale rassemblant différentes organisation de musulmans, créée en 1986. Le titre de Scheikh ül Islam est attribué par les membres du Conseil comme label honorifique à vocation interne au Conseil. Il n'est reconnu ni par les autorités allemandes, ni par les Süleymanci, ni par la DITIB. La campagne de presse et la polémique déclenchées par la révélation de ce titre en Turquie dérivent directement de la référence historique attachée à celui-ci. Il fait en effet directement allusion à une institution religieuse de l'Empire ottoman disparue avec l'avènement de la République ${ }^{69}$. Une interprétation uniquement instrumentale ne rend pas compte du caractère relationnel de la ressource - par exemple de la légitimité acquise par la carrière en Allemagne - et des modifications des valeurs qui lui sont attachées. En ce sens, les trajectoires individuelles ne sont pas isolables des contextes sociaux et les capitaux symboliques pas nécessairement convertibles d'un espace à l'autre. "En résumé et plus abruptement, ni la "valeur» ou l'efficacité des ressources politiques, ni leurs "propriétés" - aussi "intrinsèques" soient-elles -, ni les calculs ou même les manipulations dont elles sont l'objet ne peuvent être appréhendés indépendamment de leurs rapports aux contextes sociaux dans lesquels les mobilisations s'inscrivent (...) » 70

Conclusion L'utilisation du prisme transnational pour instruire la réalité de l'islam turc d'Allemagne procède d'un double constat. Le premier est d'ordre heuristique: la diversité des processus et des itinéraires que recouvre l'islam en Europe impose une reformulation des catégories de lecture face à une définition de l'islam comme une origine dont on se réclame, et une pratique plus ou moins lâche. Dans cette perspective, à l'instar de $\mathrm{P}$. Veyne dénonçant la réduction de la réalité des sentiments religieux à une analyse binaire, le transnational entendu comme espace favorisant la capitalisation , voire la reconversion de différentes ressources, sort de l'exclusivité de l'argument citoyen, accumulation de droits et d'obligations, à l'aune duquel se mesure l'intégration des jeunes issus de l'immigration ou plutôt leur «progression sur l'axe pervers de l'intégration " pour reprendre les termes de $\mathrm{J}$. Leca ${ }^{71}$. Le second constat est lié à l'apparition dans les espaces publics allemand et turc de figures individuelles " inédites » dans la mesure où, faisant le choix du politique, elles sont inattendues dans un contexte de fermeture de l'accès à la citoyenneté allemande. Nous avons vu comment des contraintes et des opportunités définies par des structures institutionnelles nationales (allemandes) ont une incidence sur les modalités organisatrices et sur le niveau de mobilisation des musulmans turcs. Les structures organisées comme les associations sont, à l'évidence, la ressource principale de la dynamique transnationale, diminuant les coûts de l'engagement pour les individus recrutés et représentant une certaine garantie de succès ${ }^{72}$. L'utilisation du cadre d'opportunité proposé par l'Allemagne ne marque toutefois pas la déconnexion d'axes 
de mobilisation enracinés dans la politique intérieure turque. Au contraire, ce sont ces opportunités qui les rendent accessibles et autorisent des jeux de réseaux, de loyauté dans un registre identitaire qui impose de lire la participation des acteurs indépendamment de leur position de citoyen ou de non-citoyen de l'État dans lequel ils résident. Ces dynamiques, ces différences d'échelles (individuelles, associatives) ne sont toutefois pas sans limite. L'investissement multisectoriel des associations et les qualités organisationnelles performantes ne doivent pas pour autant conduire à négliger le contexte et les effets de la dé-territorialisation des enjeux. Si le transnational se révèle un espace pertinent de l'action en tant qu'il permet à quelques individus de maximiser les gains d'une socialisation associative ancrée dans le référent religieux et d'entrer en politique, en Allemagne ou en Turquie, il trouve rapidement ses limites lorsque les configurations stato-nationales en reprennent le contrôle. L'exploitation par des acteurs dé-territorialisés des opportunités offertes par un environnement immédiat ne consiste finalement pas tant en une fin des territoires que dans un changement de son utilisation : de source de légitimité de l'exercice d'une souveraineté étatique, il devient lieu d'expression des identités et de construction des mobilisations, alors même que l'entrée en politique demeure in fine validée ou refusée par la configuration statonationale dans laquelle elle s'exerce.

\section{NOTES}

1. Sur le « retour du sacré » et les stratégies internationales/transnationales des acteurs religieux, voir Bertrand Badie, Marie-Claude Smouts, Le retournement du monde. Sociologie de la scène internationale, Paris, Presses de la FNSP, 1992 pp. 53-67. Sur la fluidité du religieux vis-à-vis des frontières politiques, voir Susanne Rudolf, James Piscatori (eds), Transnational Religion and Fading States, Boulder, Westwiew, 1997.

2. Derrière cette association entre identité religieuse des populations et accusation d'ingérence se profile l'idée de menace fondée sur l'opposition traditionnelle entre sédentaires et nomades. La mobilité et son contrôle, notamment au regard de l'allocation et de la redistribution de ressources par l'État, ont joué un rôle essentiel dans la mise en place des appareils législatifs réglant la situation juridique des populations étrangères. Voir à ce sujet Jan \& Leo Lucassen (ed), Migration, Migration History, History : Old Paradigms and New Perspectives, Bern, Lang, 1997.

3. Voir à ce sujet Khachig Tölölyan, «Rethinking Diaspora(s) : Stateless Power in the Transnational Moment », Diasporas, $5: 1,1996$, pp. 3-35. Les aspects transnationaux de l'immigration sont par ailleurs un volet conséquent de la littérature américaine sur le sujet. La place des réseaux dans les transactions économiques de l'entrepreunariat ethnique (acquisition d'informations et de capitaux, contraintes effectives sur les stratégies individuelles, capacité individuelle à contrôler les ressources en vertu de l'appartenance à un ou des réseaux) est ainsi au centre de l'ouvrage dirigé par Alejandro Portes (ed.), The Economic Sociology of Immigration. Essays on Networtks, Ethnicity and Entrepreneurship, New York, Russell Sage Foundation, 1995 et en 
particulier du chapitre rédigé par Mark Granovetter, «The Economic Sociology of Firms and Entrepreneurs ", pp. 128-165. Les travaux de Peggy Levitt soulignent quant à eux la fonction performative du transnational dans la mise en place, l'organisation et la structuration des objectifs de populations immigrées au niveau local. Elle insiste en particulier sur la connaissance du pays d'origine des parents qu'ont les générations qui n'y ont jamais vécu, donc au-delà de l'expérience directe, utilisant pour illustrer son propos le concept de « social remittances ", c'est-à-dire des « ideas, behaviors and social capital that flow from receiving to sending country communities ». Peggy Levitt, «Transnationalizing Community development : The Case of Migration Between Boston and the Dominican Republic ", Nonprofit and Voluntary Sector Quarterly, vol. 26, no. 4, December 1997, pp. 509-526, p. 512 ; et « Social Remittances : migration-driven, locallevel forms of cultural diffusion », International Migration Review, 1998.

4. Les travaux des historiens permettent de relativiser la «nouveauté » du label «transnational » en matière de migrations. De fait, si la sociologie des migrations, américaine comme européenne, s'en empare récemment, après 1995, les travaux historiques sur les flux migratoires au XIXème siècle font clairement état de dynamiques transnationales. Sur les « stratégies de succès » construites sur la base de cette fausse nouveauté du concept de transnational, voir Ewa Morawska, « The Sociology and History of Immigration : Reflections of a Practitioner », in Michael Bommes, Ewa Morawska (eds), Reflections on Migration research : Constructions, Omissions, and Promises of Interdisciplinarity, à paraître.

5. Cf. Saskia Sassen, The De-Facto Transnationalizing of Immigration Policy. Florence : European University Institute (RSC-Jean Monnet Chair Papers), 1996. Voir également Yasemin Soysal, Limits of citizenship : migrants and postnational membership in Europe, Chicago, University Press of Chicago, 1994.

6. Travailler sur l'appartenance religieuse impose de se donner les moyens de traiter ensemble d'une population aux degrés de pratiques multiples. Dans notre travail de terrain, nous avons fait le choix de circonscrire l'échantillon des musulmans turcs en les sélectionnant sur la base de leur appartenance formelle à l'une des trois associations quantitativement les plus représentées en Allemagne.

7. L'assimilation des musulmans à une communauté est un poncif récurrent. La notion de communauté (Umma) n'est pas usurpée et désigne bien un élément de l'énoncé du Texte (voir notamment Coran, $V, 143 / 137)$ qui s'inscrit d'ailleurs moins dans une spécificité religieuse que dans une continuité politique avec la période ante-islamique. La solidarité de la communauté est un élément fondamental du dogme religieux repris par les régimes politiques inspirés par l'islam. L'Umma n'est toutefois pas comparable encore moins assimilable à l'État en tant qu'il est défini comme exerçant sa souveraineté sur un territoire défini par des frontières. Sur ces questions, voir Montgomery Watt, Islamic Political Thought. Edinburgh, Edinburgh University Press, 1968. Dans le contexte politique d'Europe Occidentale, le terme " communauté » s'inscrit dans la tension dialectique universalisme/particularisme structurant la Nation moderne et postulant le dépassement des liens communautaires comme condition de la citoyenneté. Dans la situation actuelle de l'islam en Europe, ne serait-ce qu'au regard des diversités ethniques, la communauté des musulmans est pourtant davantage imaginée que vécue.

8. La perception des différences d'opportunités liées au contexte et de leur rôle dans les choix des acteurs fait référence aux travaux de Herbert Kitschelt et de Sidney Tarrow sur les structures d'opportunités politiques (POS), ainsi qu'à l'utilisation qu'en a fait 
Hans-Peter Kriesi à propos de ses implications pour la mobilisation des nouveaux mouvements sociaux. Hans-Peter Kriesi, The Political Opportunity Structure of New Social Movements : Its Impact on Their Mobilizisation, WZB, FS III 91-103, Berlin, Février 1991. Ces analyses se concentrent toutefois sur une interprétation fixe isolant l'appareil institutionnel de toute relation dynamique, de toute interaction avec les acteurs en mouvement, dans notre cas groupes et/ou individus mobilisés autour du référent religieux. Nous nous y référerons dans cet article dans une perspective de dédoublement des espaces d'action entre Turquie et Allemagne, et sans perdre de vue les choix tactiques des acteurs, associatifs ou individuels, le dispositif juridico-politique à partir duquel se pense traditionnellement la situation de l'immigré turc en Allemagne ne suffisant pas à instruire la réalité des divers segments biographiques dont nous rendons compte ici.

9. L'idée de citoyenneté correspond dans notre utilisation moins « au désir civique de s'engager dans le destin d'une collectivité globale à laquelle on s'identifierait (la citoyenneté comme "input" » qu'au « désir rationnel de recevoir les moyens de garantir un minimum de sécurité contre l'arbitraire d'un pouvoir extérieur (la citoyenneté comme "output") ». Jean Leca, « La démocratie à l'épreuve des pluralismes », Revue Française de Science Politique, vol. 46, n², 1996, pp. 225-279, p. 261.

10. Voir Jocelyne Césari, «Transnational Networks between Europe and Maghreb : a Risk for Nation-Sates ? », texte de l'intervention présentée à la conférence Integrating Immigrants in Liberal States, Florence, 8-9 mai 1998.

11. Ce que B. Badie appelle le retour de l'individu sur la scène internationale : « Les événements internationaux se construisent de plus en plus en fonction de l'évolution des arbitrages que consentent les individus entre leur statut de citoyen qui les lie classiquement à un territoire et leurs identités religieuse, linguistique, familiale ou micro-communautaire qui les conduisent à le transcender ou à l'ignorer ». Bertrand Badie, La fin des territoires. Essai sur le désordre international et sur l'utilité sociale du respect, Paris, Fayard, 1995, pp. 240-252.

12. A partir du cas américain, Tilly distingue entre « sending networks » et « receiving networks », soulignant l'importance d'une approche en termes de réseaux comme étant seule capable de rendre compte de la diversité migratoire. Charles Tilly, « Transplanted Networsk », in Virginia Yans-Mac Laughlin (ed.), Immigration Reconsidered. History, Sociology, and Politics, Oxford, Oxford University Press, 1990, pp. 79-95.

13. Robert O. Keohane, Joseph S. Nye, Transnational relations and World Politics, Cambridge, Harvard University Press, 1972.

14. Sur cet aspect, les travaux de Czarina Wilpert demeurent la référence, Czarina Wilpert, Ali Gitmez, «A Micro-society or an Ethnic Community ? Social Organisation and Ethnicity amongst Turkish Migrants in Berlin ", in John Rex, Daniele Joly, Czarina Wilpert (eds), Immigrants Associations in Europe, Aldershot, Grower, 1987, pp. 86-125. 15. Ariel Colonomos, "L'acteur en réseau à l'épreuve de l'international ", in MarieClaude Smouts, Les nouvelles relations internationales. Pratiques et théories, Presses de Sciences-po, Paris, 1998, pp. 203-226. Et également Ariel Colonomos (dir.), Sociologie des réseaux transnationaux, Paris, L'Harmattan, 1995.

16. Suivant le travail de définition de l'objet religieux effectué par Danièle HervieuLéger dans La religion pour mémoire, Paris, Le Cerf, 1986.

17. James N. Rosenau, The study of global interdependence : Essays on the transnationalism of world affairs, Londres, Pinter, 1980. 
18. Le vote des Turcs vivant à l'étranger représente plus de 3 millions de personnes qui doivent exprimer leur suffrage sur le sol national, et non dans les consulats étrangers comme le peuvent d'autres populations étrangères résidant en Europe (cf. les

Algériens). En 1995, Ankara a fait quelques efforts : des urnes ont été installées dans les aéroports en Turquie comme pour faire la moitié du chemin, considérant les aéroports internationaux comme des frontières. Ne sont partis voter que ceux qui pouvaient en assumer la charge financière. « Ob der Weg zu unserer Wohlfahrt in Deutschland über das Mutterland der rechte Weg ist ? ", Perspektive, février 1996, p. 47.

19. Non seulement les protagonistes changent de nature, mais leurs objectifs et moyens d'action sont radicalement nouveaux et différents de ceux des gouvernements. A la recherche non de la conquête des territoires mais de l'accès à la négociation, moins visibles, quelquefois clandestins : « Ils visent moins l'Avoir que la Reconnaissance ». Didier Bigo, « Nouveaux regards... », op. cit., pp. 336-7.

20. « Regular interactions across national boundaries when at least one actor is a nonstate agent or does not operate on behalf of a national government or an intergovernmental organization ». Thomas Risse, «Bringing transnational relations back in : introduction ", in Thomas Risse (ed.), Bringing Transnational Relations Back In, Cambridge, Cambridge University Press, 1995, p. 3.

21. Créée en mars 1924, la Diyanet Isleri Türkiye Islam Birligi, Bureau des Affaires Religieuses de l'Etat noté DITIB, sans renier la dimension identitaire de l'islam pour la société turque, notamment dans ses valeurs et sa force d'identification au plan de la Nation, promeut toutefois un certain islam, non traditionnel, aux normes du projet d'occidentalisation dont la sécularisation est la traduction. La DITIB gère les rapports entre islam et laïcité au coeur du projet kémaliste : «(...) the hub of the Westernization was thus secularization and the hub of secularization was the re-definition of Islam in accordance with the new purposes of the State ». Voir à ce sujet le remarquable travail de Menderes Çinar, The Republican Character of Islamism in Turkey from the perspective of the Political, PhD in Philosophy in Political Science and Public Administration, Bilkent University, Ankara, Décembre 1998, p. 201.

22. Aujourd'hui Fazilet Partisi, Parti de la Vertu.

23. Ce contrôle de "l'islam kémaliste » est placé, officieusement mais de plus en plus explicitement, sous l'autorité de l'institution militaire en Turquie. En février 1997, la réunion du Conseil National de Sécurité fixe le cadre politique légitime dans lequel les islamistes du Refah, membre de la coalition gouvernementale Refahyol, sont sommés d'évoluer. Les limites imparties prennent en compte la dimension religieuse en réprimant notamment les imam hatip lisesi, lycées de formation des imams. Autre phénomène sur lequel l'institution militaire plaque son contrôle : la construction des lieux de culte.

24. Il ne s'agit pas du seul registre à propos duquel l'Etat turc maintient active sa gestion des intérêts nationaux. Dans l'espace de circulation et de mobilité des pratiques transnationales, l'État devient donc un acteur parmi d'autres, aux côtés de réseaux, d'individus, restés en Turquie ou vivant en Allemagne[[Au radicalisme d'un J. Rosenau dénonçant la prison paradigmatique du stato-centré, succède l'idée que le transnational n'est pas une alternative à l'action d'Etat mais que les deux dynamiques interagissent.

25. Henri Lefebvre, La production de l'espace, Paris, Antrhopos, 1974, p. 19.

26. Voir Didier Bigo, « Nouveaux regards sur les conflits?», in Marie-Claude Smouts, Les nouvelles relations internationales, op. cit., pp. 309-354, p. 336. Et aussi « Guerres, 
conflits, transnational et territoire ", Cultures et Conflits, nº21/22, printemps-été 1996, pp. 397-418.

27. Nous considérons ici l'Etat à l'aune des différents registres à travers lesquels il s'exprime : territorial, national, participatif et providence. Voir Stein Rokkan, « Dimensions of State Formation and Nation-Buidling », in C. Tilly (ed.), The Formation of National States in Western Europe, Princeton, Princeton University Press, 1975, pp. 562-600.

28. Le travail politique de proximité, en Allemagne comme en Turquie, est le comparative advantage principal des groupes islamiques, en particulier souligné par la presse turque au moment des campagnes municipales et législatives dont le Refah est sorti vainqueur.

29. Bertrand Badie, La fin des territoires, op. cit.

30. C'est-à-dire de structures de compréhension ayant donné forme à l'expérience dans un système politique particulier. En ce sens, la culture politique ne s'entend pas de manière autonome mais se situe avant tout historiquement. Le principe de territorialité (comme convention, moyen de contrôle sur une population, délimitation d'une communauté politique) est produit lui aussi d'une culture particulière. Bertrand Badie, Culture et politique, Paris, Economica, 1993.

31. Le mimétisme est un comportement clef des associations islamiques, jouant sur la légitimité des répertoires d'action et de revendication usités pour accroître leur capacité performative. Voir Stephen D. Krasner, « Power politics, institutions, and transnational relations ", in Thomas Risse, op. cit., pp. 257-279.

32. Opportunités au sens de "consistent -but not necessarily formal or permanentdimensions of the political environment that provide incentives for collective action by affecting people's expectations for success or failure". Sidney Tarrow, Power in Movement. Social Movements and Contentious Politics, Cambridge University Press, 1998, p. 76-77.

33. Cela se vérifie pour d'autres groupes de populations. L'exemple kurde est par exemple analysé par Hamit Bozarslan, La question kurde : Etats et minorités au MoyenOrient, Paris, Presses de Sciences-Po, 1997.

34. Voir Michael Bommes, « Die Beobachtung von Kultur. Die Festschreibung von Ethnizität in der bundesdeutschen Migrationsforschung mit qualitativen Methoden », in Jahrbuch für Soziologiegeschichte 1994, Opladen, Leske+Budrich, 1996, 205-226.

35. Sidney Tarrow, op. cit., p. 81.

36. La définition de l'Etat allemand comme semi-souverain développée par Peter Katzenstein d'un côté, et la valeur légitimante de l'institution et du constitutionnalisme soulignée par R. M. Leipsius de l'autre, permettent de cerner la forte imprégnation de la culture politique allemande par le droit, dimension que l'on retrouve dans le débat juridique sur les étrangers. « (...) l'interaction entre politiques publiques (policy) et vie politique (politics) est affectée prioritairement par l'organisation institutionnelle au pouvoir plus que par les impératifs d'action inhérentes aux problématiques politiques ». Peter Katzenstein, Policy and Politics in West-Germany : The Growth of a Semi-sovereign State, Philadelphie, Temple University Press, p. 7. Rainer M. Lepsius, Interessen, Ideen und Institutionen, Opladen, Westdeutscher Verlag, 1990.

37. «Path dependence means that history matters ", Douglas C. North, Institutions, Institutional Change and Economic Performance, Cambridge University Press, 1990, p. 100. 
38. La gestion europénne de l'islam passerait par une nécessaire régulation par une institution représentative des intérêts de tous les musulmans. Le problème est énoncé et lu en ces termes dans l'ensemble des États européens, avec dans certains cas comme en Belgique, la concrétisation de cet objectif.

39. Helmut Kohl, Discours du 6 juillet 1997 à l'occasion du 50ème anniversaire de l'Académie protestante de Tutzing.

40. Jean-Yves Carlier, Michel Verwilghen (dir.), Le statut personnel des musulmans. Droit comparé et droit international privé, Bruxelles, Bruylant, 1992. Voir en particulier dans cet ouvrage l'article de Albert Bastenier, « La question sociologique : la régulation étatique de l'islam dans trois contextes européens », pp. 13-20.

41. «Pourquoi les a-t-on appelés des Gastarbeiter? Parce qu'on ne pouvait pas les appeler des Fremdarbeiter, étant donné que c'était le nom des travailleurs pendant la Seconde Guerre mondiale. On a donc essayé de trouver quelque chose de gentil : on les accueillera comme des hôtes et, à côté, ils travailleront un peu, ce sera bon pour tout le monde. Mais derrière cette conceptualisation, il y avait, de la part de la société allemande, l'idée fondamentale qu'ils retourneraient chez eux ». Daniel Cohn Bendit, « Les politiques de l'immigration en Allemagne », in François Dubet, Michel Wieviorka (dir.), Penser le sujet, Paris, Fayard, pp. 589-601.

42. Véritable signe ostentatoire du déclenchement de la manoeuvre militaro-politique qui aboutira à la chute du gouvernement Refahyol regroupant islamistes du Refah et conservateurs du DYP, en février 1997, le Conseil National de Sécurité publie une liste de mesures-avertissements à l'adresse des islamistes, fixant le cadre légitime d'exercice $\mathrm{du}$ politique en Turquie tel que conçu par l'institution militaire. L'ultime mesure pointe le transnational en interdisant le financement des partis politiques turcs par des « organisations installées en Europe à l'instar de Milli Görüs ».

43. P. Levitt en donne la définition suivante : « the ability to effectively articulate and achieve organisational goals ». Peggy Levitt, « Transnationalizing Community Development : The Case of Migration Between Boston and the Dominican Republic », vol. 26, n 4, dec. 1997, pp. 509-526, p. 510.

44. Le changement intervient lors de la publication en 1997 d'un ouvrage de W. Heitmeyer (Wilhem Heitmeyer, Joachim Müller, Helmut Schröder, Verlockender Fundamentalismus, Francfort, Suhrkamp, 1997) fortement critiqué même si depuis lors désigné comme référence par les médias et l'opinion publique. Voir à ce sujet la critique bibliographique de Nicola Tietze, Cultures et Conflits, nº31-32, automne-hiver 1998, pp. 233-237.

45. La référence en matière quantitative demeure le recensement de 1987, même si quelques régions ont passé commande de ce type d'études. C'est le cas des villes de Berlin, Hambourg, de la région de Rhénanie du Nord Westphalie.

46. Pour une critique bibliographique plus développée, voir Valérie Amiraux, Itinéraires turcs musulmans d'Allemagne. Appartenances religieuses et modes d'intégration, Thèse pour le doctorat en science politique, IEP Paris, 1997.

47. Même si les grandes formations politiques -SPD, CDU, FDP en particulier- ont organisé leur groupe de réflexion sur les « Turcs d'Allemagne » par les « Turcs d'Allemagne ». Cem Özdemir et Leyla Onur, deux députés du Bundestag d'origine turque, en ont longtemps été les illustrations. En outre, l'exportation de la violence politique et des affrontements turco-turcs sur le sol allemand est au centre de la problématisation politique des relations bilatérales Turquie-Allemagne. 
48. Valérie Amiraux, « Jeunes musulmanes turques d'Allemagne. Voix et voies de l'individuation ", in Felice Dassetto (dir.), Paroles d'islam, Paris, Maisonneuve et Larose, 1999.

49. Danièle Hervieu-Léger, « Renouveaux » religieux et nationalistes : la double dérégulation » in Pierre Birnbaum (dir.), Sociologie des nationalismes, Paris, P.U.F, 1997, pp. 163-185.

50. Voir le numéro spécial de la revue Cultures en mouvement, n 13, décembre 1998/ janvier 1999, pp 25-51, et les ouvrages particuliers des auteurs des articles du numéro. 51. Olivier Roy, « Naissance d'un islam européen », Esprit, janvier 1998, pp.10-35.

52. En particulier pour désigner une type particulier de musulmans, à savoir ceux « qui, sans toujours se conformer aux règles du culte, considèrent la référence islamique comme une partie de leur héritage familial et culturel ». Jocelyne Césari, Musulmans et Républicains. Les jeunes, l'islam et la France, Paris, Complexes, 1998, p. 41.

53. Ulf Hannerz, Cultural complexity : studies in the social organization of meaning, New York, Columbia University Press, 1992.

54. Bertrand Badie souligne dans la conclusion de son ouvrage l'inéluctable parallèle entre le retour sur l'individu et la croissante importance des réseaux. B. Badie, La fin des territoires, op. cit.

55. B. Badie, op. cit., p. 240.

56. Ayse Caglar, « German Turks in Berlin : social exclusion and strategies for social mobility », New Community, 21(3), July 1995, pp. 309-323, p. 320.

57. Longtemps principales ressources financières pour les associations et les formations politiques car fournissant un bénéfice économique immédiat et symbolique à moyen terme, ces activités de nature communautaire ont également pu servir de tremplins à des vocations politiques individuelles. La presse allemande en fait d'ailleurs depuis quelques années l'indicateur de réussite principal des Turcs en Allemagne, là où son homologue français traquait le nom à consonnance « beur " pour signifier l'intégration des jeunes d'origine maghrébine dans le jeu politique français. Dans le cas allemand, la presse se fait l'écho de deux types de réussite, d'une part inspirée du modèle « Immigrün » s'inspirant du cas de Cem Özdemir, député Vert d'origine turque élu au Bundestag, et d'autre part de la réussite de l'ethnic business, en particulier dans l'alimentaire et essentiellement dans le commerce du Döner. Pour un article de synthèse sur l'ethnic entrepreneurship, voir Jean-Pierre Cassarino, The Theories of Ethnic Entrepreneurship, and the Alternative Arguments of Social Action and Network Analysis, EUI Working Papers, Florence, SPS No.97/1.

58. Le contrôle par une hiérarchie centrale installée en Turquie est une donnée fondamentale de la qualité de l'échange transnational et il n'est pas question, pour les trois formations qui nous occupent, de laisser se détacher un élément satellite.

59. Source : Informationsdient, 1/94, p. 6.

60. Voir Valérie Amiraux, « Transnational en puissance, transnational en acte. Le rôle de l'espace migratoire dans la mobilisation politique islamiste », in G. Groc (dir.), L'Autre Islam (« Formes nouvelles de l'islam en Turquie »), Revue de l'ERISM-INALCO, à paraître 1999.

61. Il s'agit du DTP (Demokratik Türkiye Partisi), Parti Démocratique de Turquie.

62. L'Avrupa Milli Görüs Teskilatlari (AMGT), rebaptisée Islamische Gesellschaft Milli Görüs (IGMG) en 1995, s'installe en Allemagne sous un autre label en 1972. Sunnite, l'association est quantitativement la plus représentée en Allemagne en termes de lieux de prières, cours coraniques et nombre de militants et sympathisants, après la Diyanet 
(DITIB), délégation du Bureau des Affaires Religieuses turc. Voir Valérie Amiraux, « Islam turc d'Allemagne. Les dynamiques de l'identité religieuse », in Rémy Leveau (dir.), Islam(s) en Europe, Berlin, Les Cahiers du Centre Marc Bloch, 1998, pp. 17-49. 63. La mise en place des Conseils d'étrangers correspond, au milieu des années soixante-dix, à la prise de conscience qu'un certain nombre de besoins et de demandes des populations étrangères ne sont pas prises en compte, en même temps qu'elle entre dans le jeu politique allemand consistant à gagner des voix allemandes en flattant les étrangers. A vocation informative et consultative, ils sont conçus comme lieux d'expression des minorités non allemandes, la fonction évoluant par la suite de conseil auprès des affaires municipales à la représentation des intérêts des étrangers. Essentiellement fréquentés par les populations espagnoles, portugaises et italiennes jusqu'à l'entrée en vigueur du Traité de Maastricht, la tendance y a longtemps été celle des listes par nationalité mais, dans le Land de Rhénanie du Nord Westphalie par exemple, depuis le début de la décennie quatre-vingt-dix, des listes d'union islamique, rassemblant différents visages de l'islam d'Allemagne sont arrivées premières aux élections des Conseils d'Etrangers.

64. Pour l'utilisation dans le champ de l'économie politique, voir Warren F. Ilchman, Norman Thomas Uphoff, The political Economy of Change, Berkeley, University of California Press, 1969.

65. La représentation est entendue au sens de la construction symbolique permettant aux individus de se mobiliser.

66. Sur les questions de dévaluation du capital, notamment culturel, rencontrés par les Turcs rentrés au village, et sur les moyens de compensation mis en place pour recouvrer des positions de choix dans la société d'origine voir Barbara Wolbert, « Rückkehr : Statuspassage und Passageriten türkischer Migrantinnen », Zeitschrift für Ethnologie, 115 (1990), pp. 169-197.

67. Voir Amitai Etzioni, The active society : a theory of societal and political processes, Londres, Collier-Macmillan, 1968. M. Dobry plaide quant à lui pour une définition plus étroite des mobilisations considérés uniquement dans la perspective de l'existence d'une ligne d'action et dans un cadre conflictuel. Voir Michel Dobry, Sociologie des crises politiques : La dynamique des mobilisations multisectorielles, Paris, Presses de la FNSP, 1986.

68. Voir à ce sujet l'article d'A. Caglar précédemment cité et notamment son illustration à partir de la recherche et de l'acquisition d'un logement, en Turquie comme en Allemagne. Si un turc d'Allemagne peut se permettre financièrement d'acquitter un loyer hors de prix, ni son nom, ni son passeport ne lui permettent un accès libre au marché du logement.

69. Le personnage titulaire de ce titre était le seul autorisé à se tenir debout en présence du Sultan. Sur cette polémique, voir l'ouvrage de Oral Calislar, Refah Partisi : Nereden, Nereye ?, Istanbul, Pencere Yayinlari, 1995.

70. Michel Dobry, op. cit., p. 38.

71. Jean Leca, « La démocratie à l'épreuve des pluralismes », op. cit., p. 262.

72. Bert Klandermans, Sidney Tarrow, « Mobilization into social movements : synthesizing European and American approaches », International Social Movement Research, vol. 1, 1988, pp. 1-38. 
INDEX

Mots-clés : mobilisation, Relations Internationales, réseaux transnationaux, Mondialisation

\section{AUTEUR}

VALÉRIE AMIRAUX

Jean Monnet Fellow, Institut Universitaire Européen, Florence. 